\title{
EFFECT OF DIFFERENT TYPES OF POLYMERIC MICROCAPSULES ON THE SELF-HEALING EFFICIENCY OF CEMENT BASED COMPOSITES
}

\author{
ANTONIOS KANELLOPOULOS", CHRYSOULA LITINA ", PETROS GIANNAROS" AND \\ ABIR AL-TABBAA* \\ University of Cambridge \\ Department of Engineering \\ Cambridge, UK \\ e-mail: $\underline{\text { ak880@cam.ac.uk }}$
}

Key words: Polymeric microcapsules, self-healing, fracture, durability recovery

\begin{abstract}
Several different healing agents and mechanisms have been put forward for use in autonomous healing of small cracks in cementitious composites. While the existing research on this topic focuses mainly on the use of polymeric materials as encapsulated healing agents, this study aims at assessing the efficiency of inorganic silica precursors as potential healing materials. This work investigated the effect on self-healing of different types of polymeric microcapsules containing silica precursors. The microcapsules were embedded in three different types of cement-based matrix, namely mortar, paste and grout. The results in all cases showed that the inclusion of microcapsules resulted in a marginal reduction of compressive strength in more cases for low volume fraction additions. Increasing the microcapsules concentration increased the reduction in the compressive strength. However, the observed crack healing $\%$ and durability recovery were substantially larger than any reduction on the mechanical properties. In addition, the incorporation of polymeric microcapsules in the cementitious matrix resulted in slight improvement of the composites' fracture toughness.
\end{abstract}

\section{INTRODUCTION}

Amongst the self-healing techniques developed in the last twenty years the microencapsulation approach is by far the most studied. Microencapsulation was initially developed for self-healing applications in polymers and composites [1] and developed from the previous systems based on hollow capillary tubes [2]. The two techniques have many similarities, but the use of microcapsules alleviates the manufacturing related issues associated with the incorporation of hollow tubes in matrices.

In principle, microcapsules are containers that envelope a healing compound keeping it protected from the manufacturing processes as well as from the surrounding host matrix. The most fundamental principle of self-healing via microencapsulation is that the microcapsules are homogeneously dispersed in the bulk volume of the host material and the release of their healing compound is triggered by the formation of cracks that rupture their shell. Consequent chemical interactions between the encapsulated material(s) and the host matrix heal the crack restoring partially or fully the bulk material properties.

Since the microcapsules are additions within the bulk volume of the host matrix it is expected they will alter its mechanical properties. The degree of this change depends on a large number of parameters: the size and the volume fraction of microcapsules, the mechanical properties of the shell materials and the 
mechanical interlock between the microcapsules and the surrounding matrix. In addition the extent of self-healing depends on four major factors: the type of the healing compound used, the size of the crack, the size of the microcapsules and consequently their volume fractions. In cases where an activator is needed to promote healing, the quality, the particle size and the concentration of the activator also play an important role. It is therefore apparent that the ideal self-healing material should have an optimised balance between the loss of its original properties, due to the inclusion of microcapsules, and the potential self-healing efficiency.

In the field of construction materials, the concept of introducing microcapsules for selfhealing is relatively new. The earliest reported studies were conducted by Pelletier and Bose [3] and Yang et al [4] for the production of selfhealing concrete, while more recently the development of microcapsules for use in bituminous materials was also reported [5]. The vast majority of articles in the field mainly deal with production methods, characterisation and survivability issues and in the best case report some preliminary healing results. Only a very limited number of studies have addressed the issue of how microcapsules affect the fracture properties of the host matrix as well as their effect on crack formation and subsequent healing.

Our research is part of a major consortium ("Materials for Life") which investigates multiscale healing on cement-based materials [6]. This paper reports on the development and performance of a discrete system of polymeric microscopic carriers, namely microcapsules, to deliver and release a silica-precursor repair solution for cementitious materials. Previous work in our group has confirmed the potential of both liquid and powder minerals for use in self-healing cement-based composites $[7,8]$. The effect of different polymeric microcapsules, carrying sodium silicate or a silica sol, was investigated hardened properties (compressive and fracture toughness). Furthermore, the healing potential of the used microcapsules under different cracking regimes was confirmed.

\section{MICROENCAPSULATION SYSTEMS}

Different types of microcapsules were investigated for the delivery of both, liquid and powder mineral healing agents as well as their potential to promote self-healing. Table 1 summarizes the characteristics of the different polymeric microcapsules used in this study. Two polymeric shell types were considered, gelatin and polyurea due to their extended use in microcapsule synthesis. Commercial gelatin and polyurea shelled microcapsules were manufactured in collaboration with Lambson Ltd and THIES Technology respectively, to envelope a sodium silicate core.

Additionally a prototype polyurethane system was conceived and developed within our facilities for the delivery of an aqueous silica suspension. The investigated microcapsules covered a wide range of sizes from 40 to $700 \mu \mathrm{m}$, to target different crack widths. Optical microscope images of the investigated microcapsules are presented in Fig. 1 and Fig. 2. Moreover the effects of the method of introduction of microcapsules in the cementitious host were investigated; either as part of the dry powder constituents or incorporated in the mixing water.

Table 1: Characteristics of the microcapsules used in this study

\begin{tabular}{|c|c|c|c|c|}
\hline Supplier & Notation & Shell & Cargo & $\begin{array}{l}\text { Av. } \\
\text { Size } \\
(\mu \mathrm{m}) \\
\end{array}$ \\
\hline \multirow{2}{*}{ Lambson } & L1 & \multirow{2}{*}{$\begin{array}{l}\text { Pig } \\
\text { gelatin/ } \\
\text { Gum } \\
\text { acacia }\end{array}$} & \multirow{2}{*}{$\begin{array}{l}\text { Sodium } \\
\text { silicate } \\
\text { emulsion } \\
\text { (42\% wt. } \\
\text { in oil }\end{array}$} & 700 \\
\hline & L2 & & & 300 \\
\hline THIES & $\mathrm{TH}$ & Polyurea & $\begin{array}{c}\text { Sodium } \\
\text { silicate } \\
\text { semi- } \\
\text { crystalline }\end{array}$ & 129 \\
\hline Prototype & PU & $\begin{array}{l}\text { Polyuria- } \\
\text { urethane }\end{array}$ & $\begin{array}{c}\text { Silica } \\
\text { solution } \\
\text { (40\% wt. } \\
\text { in water) }\end{array}$ & 41 \\
\hline
\end{tabular}




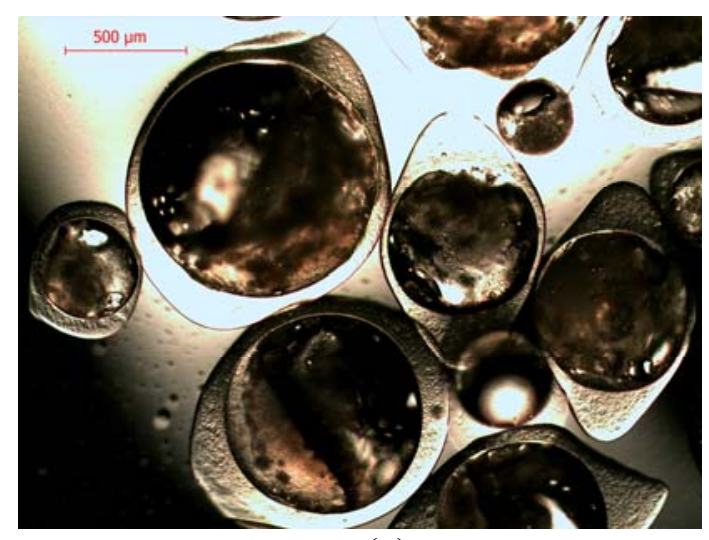

(a)

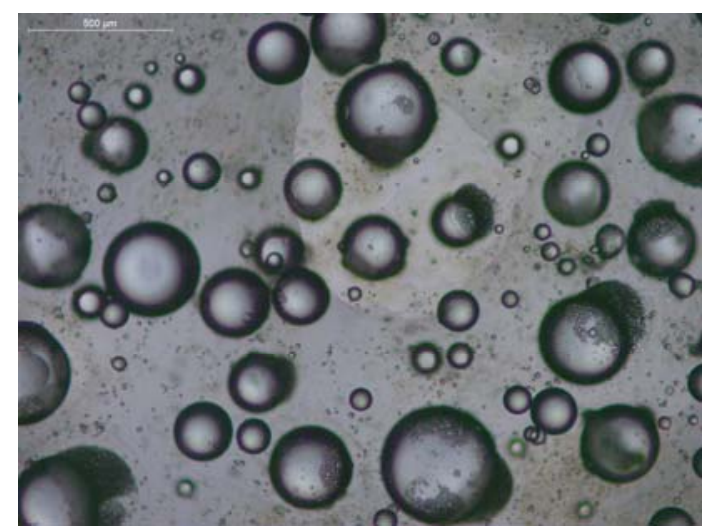

(b)

Figure 1: The types of LMB microcapsules with average size of (a) $700 \mu \mathrm{m}$ and (b) $300 \mu \mathrm{m}$.

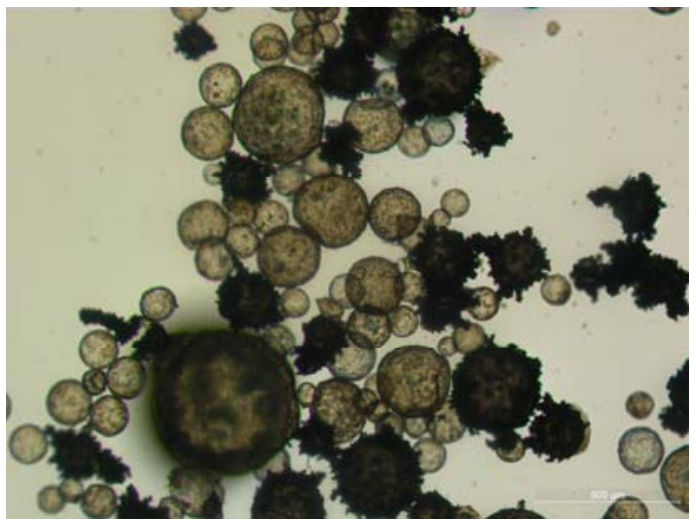

(a)

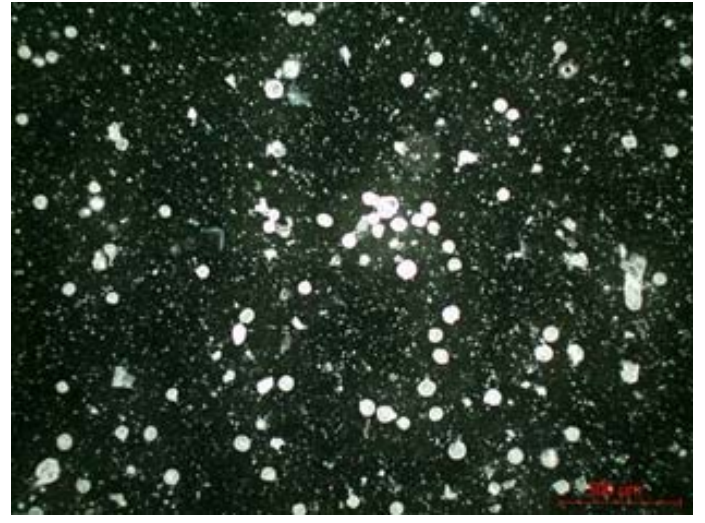

(b)

Figure 2: (a) Thies polymeric microcapsules and (b) prototype PU microcapsules.

\section{PREPARATION OF COMPOSITES AND EXPERIMENTAL METHODS}

\subsection{Materials and preparation}

The effect of different content fraction of polymeric microcapsules on the fresh, hardened and self-healing properties was investigated using both cement pastes, grout and mortar mixes. A range of microcapsule fractions in terms of volumetric content were considered. For the prototype system, two different volume percentages were considered, 4 and $16 \%$ vol. to represent a lower and higher boundary. A more detailed optimization study was performed for the Lambson microcapsules.

Concurrently different water-to-cement ratios were considered, ranging from 0.4 to 0.6 to better emulate field applications and cover a wider range of cementitious materials. For consistency all mixes were prepared using ordinary Portland cement (CEM-I, 52.5N). Pastes were prepared by dry mixing cement and free flowing microcapsule powder to ensure homogeneous dispersion before the addition of water. On the other hand mortars were produced using locally sourced fine sand, with maximum aggregate size of $1 \mathrm{~mm}$. The cementto-sand ratio was kept constant to 1.5 . The polymeric microcapsules were dispersed in the water of the mix to ensure better dispersion and distribution during mixing. Once the dry mixing was completed the solution of water and microcapsules was added in three parts into the mixer.

Following the completion of mixing the 
material was moulded in prisms (40x40x160mm), cubes $(100 \times 100 \mathrm{~mm})$ and cylinders $(100 \times 200 \mathrm{~mm})$ necessary for the experimental work. Light reinforcement (internal steel wire) was placed on the compression zone of the prisms to prevent abrupt damage of samples during and after loading. Samples were taken out of the moulds after 24 hours and then stored in water $\left(20^{\circ} \mathrm{C} \pm\right.$ $1{ }^{\circ} \mathrm{C}$ ) till testing time. To investigate the effect of degree of hydration on the healing efficiency of the proposed healing mechanism different ages were considered. Mechanical tests were performed at 1, 7 and 28 days and the samples designated to self-heal were returned to water for further 28 days.

\subsection{Influence of microcapsules on mechanical and fracture properties}

The mechanical loading of all the prisms in this study was performed on a $30 \mathrm{kN}$ static testing frame. Prior to cracking, all specimens were notched with a rotating diamond blade. The notch depth and width were 1.5 and $2.0 \mathrm{~mm}$ respectively. A clip gauge was attached close to the notch edges to monitor crack mouth opening displacement (CMOD). The prisms were loaded over a length of $125 \mathrm{~mm}$, at a rate of $0.125 \mathrm{~mm} / \mathrm{min}$. Upon load removal the residual crack widths were in the range of $0.11 \mathrm{~mm}$ (for pastes) to $0.25 \mathrm{~mm}$ (for mortars).

Compressive strength and modulus of elasticity measurements were performed on a $2000 \mathrm{kN}$ servohydraulic compression frame using 100x100mm cubic specimens and 100x200mm cylinders respectively.

\subsection{Crack healing and durability recovery}

The bottom crack faces in all the specimens were monitored over time using a stereoscope. Digital images were captured at the crack face. Cracks were photographed after cracking and at the end of the healing period. Image analysis software (Image-J) was then used to analyze the acquired captions and quantify the total crack area and area recovery (crack mouth healing$\mathrm{CMH} \%$ ). Crack mouth healing was calculated using the following formula:

$$
\operatorname{CMH}(\%)=\frac{A_{i}-A_{h}}{A_{h}} \times 100
$$

where $\mathrm{Ai}$ is the crack area on the day of cracking and $\mathrm{Ah}$ is the area at the same point at the end of the healing period.

Similarly to assess the sealing efficacy of the recovered cracks the liquid capillary absorption coefficient was estimated.

\section{RESULTS AND DISCUSSION}

\subsection{Effect of microcapsules on mechanical properties}

Figure 3 shows the effect of different volumetric additions, with respect to cement, on the compressive strength of mortars and pastes.

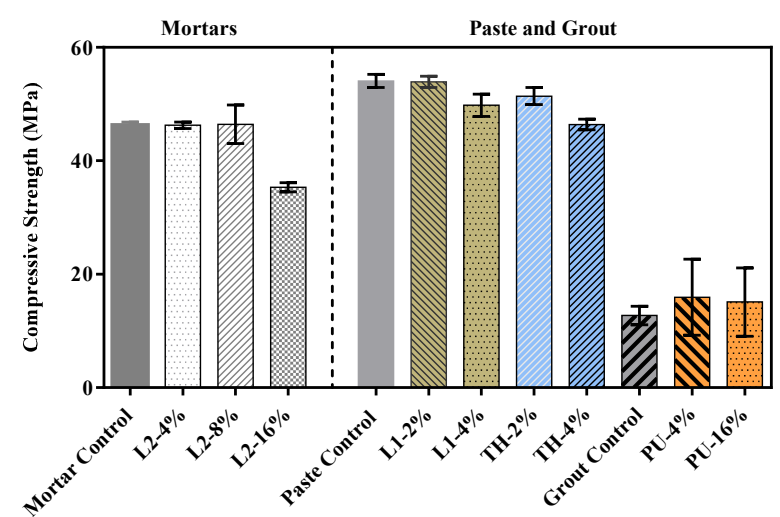

Figure 3: Effect of volumetric content of different types of microcapsules on the compressive strength.

For both types of mixtures, mortars and pastes, low volume fractions of microcapsules do not effect significantly the compressive strength of the composite. For microcapsules up to $300 \mu \mathrm{m}$ in mortars (L2) the effect on the compressive strength is almost negligible up to $8 \%$ addition. However that effect seems dependent on the type of added microcapsule and the volumetric content. Lambson microcapsules have more flexible shells whereas THIES and PU are increasingly rigid. The discrepancy also reflects the size variation of the microcapsules. However up to $8 \%$ $(\sim 1.57 \% \mathrm{Wt}$. of cement) concentration the effect seems negligible confirming previously reported strength reduction in the range of 10$15 \%$ for up to $2 \%$ wt. microcapsules $[9,10]$. 
Above $12 \%$, regardless of volume fraction the reduction in compressive strength fluctuated between $24-27 \%$.

The effect of the addition of microcapsules on the elastic modulus is illustrated in Figure 4.

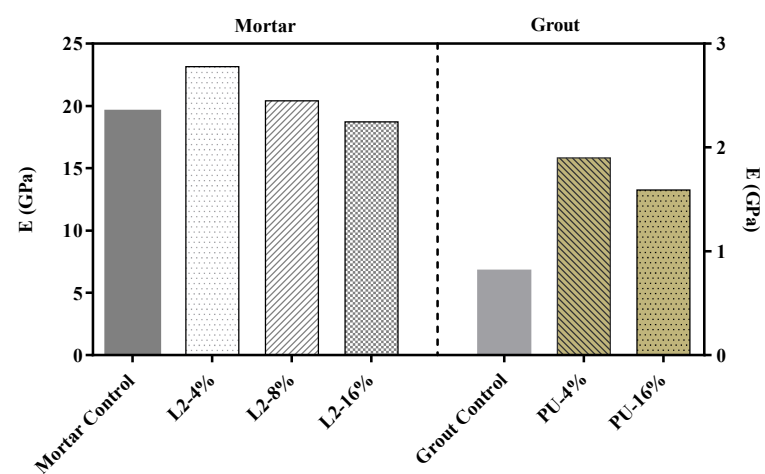

Figure 4: Effect of volumetric content of different types of microcapsules on the elastic modulus.

Addition of microcapsules showed some improvement on the measured values of the modulus of elasticity. However, increasing volume fraction of microcapsules resulted in gradual decrease in the obtained values. This observation was consistent for both mortar and grout samples. The results do not show a clear pattern on the effect of microcapsules. Interestingly the only two studies in this field that studied the effect of microcapsules on Young's modulus also reported similar fluctuations[11,12].

\subsection{Fracture toughness}

Fracture toughness variation with various volume fraction additions for different microcapsules is illustrated in Figure 5.

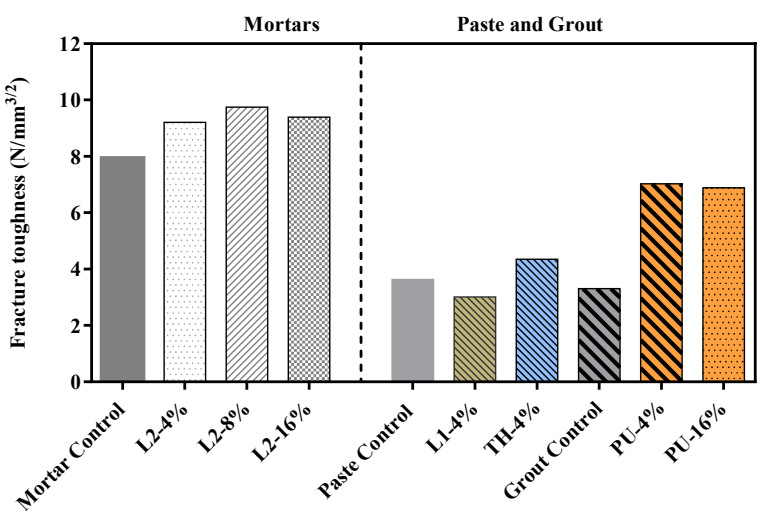

Figure 5: Fracture toughness variation with different volume fractions for mortars and cement pastes.
It is clear that the addition of microcapsules to some extent has provided an increase in the stiffness of the specimens. However, no significant changes are observed between the different volume fractions. Similarly, the incorporation of microcapsules has increased the fracture toughness. This observation is consistent with reported data on similar size polymeric microcapsules embedded in epoxy matrices [13]. Fracture toughness was found to increase for all different types of microcapsules used in this study with the exception of microcapsules L1. The relatively large size of L1 microcapsules is the reason for the marginal decrease in the obtained fracture toughness values.

Preliminary SEM observations of cracked faces revealed that microcapsules were attracting the cracks. In the vast majority of cases this attraction resulted in the deflection of the crack path and the formation of microcracks. Black arrows on Figure 6a indicate the crack paths for each case, whereas the schematic (Figure 6b) illustrates the concept.

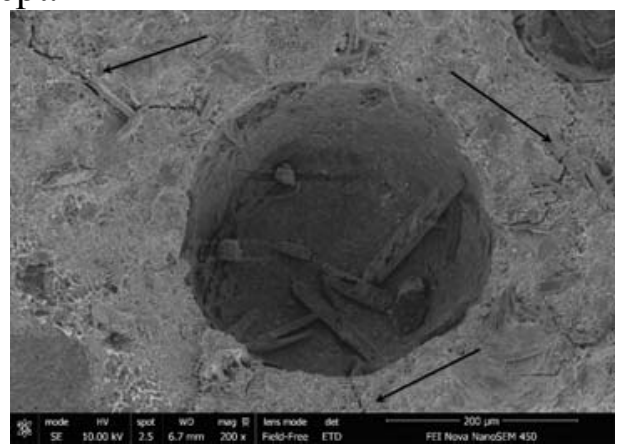

(a)

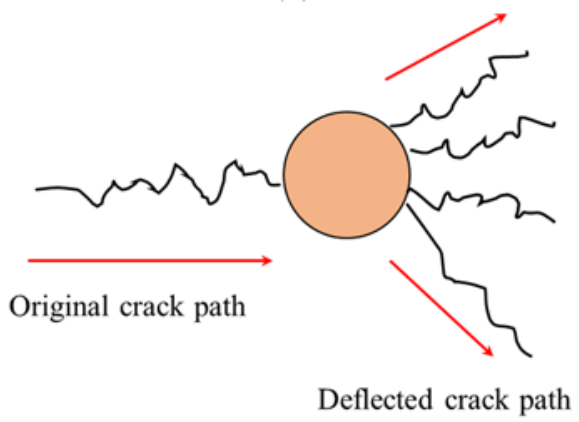

(b)

Figure 6: SEM image and schematic showing the (a) crack propagation and (b) deflection pattern around the microcapsules. 


\subsection{Crack mouth healing}

Figure 7 shows the crack mouth healing (\%) measurements performed in this study.

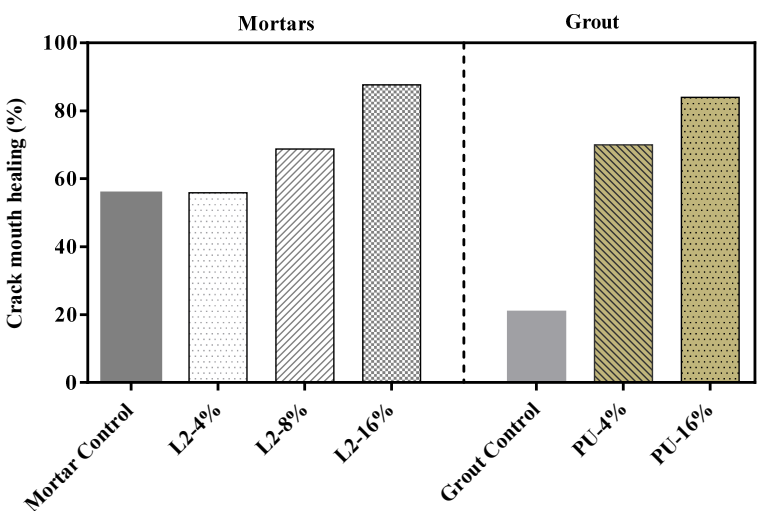

Figure 7: Crack mouth healing (\%) for different types of cementitious composites containing different volume fractions of microcapsules.

All specimens containing microcapsules, in both mortars and grouts, have healed better compared to the corresponding control samples. Although at low volume fractions (4\%) the behaviour between different composites and microcapsules is not the same at large microcapsules concentrations (16\%) the measured $\mathrm{CMH} \%$ is almost identical.

\subsection{Sorptivity}

Figure 8 shows the capillary sorption measurements for cracked samples after 28 days of healing.

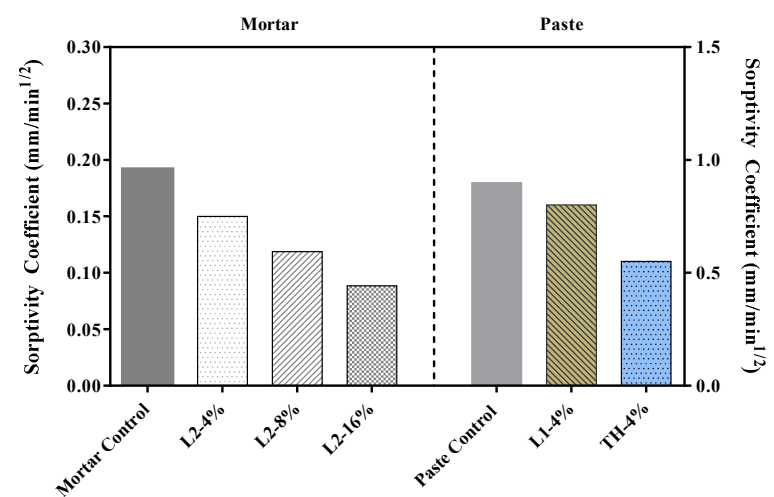

Figure 8: Sorptivity of healed samples containing different types and volume fractions of microcapsules.

Following a similar trend to $\mathrm{CMH} \%$, capillary sorption of healed samples containing microcapsules is substantially improved compared to the control samples. The formed healing products have densified into the crack blocking the water movement. For mortars, the preliminary data shows that increasing volume fraction of microcapsules results in gradual decrease of the permeability of samples. Microstructural analysis of the formed healing products has shown that they are mainly result of hydration reactions triggered by the interaction of the microcapsules cargo compounds with the host cementitious matrix $[7,10]$.

\section{CONCLUSIONS}

This study demonstrated the potential of using a microencapsulation system to recover the initial functionality of concrete structures and prolong its useful life-span. There seems to be a correlation between mechanical strength and microcapsule content, suggesting that the selection of the optimum microcapsule content depend on a number of parameters. Regardless of the type of the microcapsule and the type of the host matrix, in the vast majority of cases the incorporation of microcapsules improved the stiffness of the samples. Fracture toughness found to increase slightly compared to the control samples. The healing levels as measured by macroscopic methods showed in all cases that the inclusion of microcapsules improved the crack closure and recovered the sealing efficacy significantly. These findings were very consistent in samples initially fractured at small crack sizes. Optical and scanning electron microscopy revealed that the vast majority of microcapsules survive mixing and rupture during cracking.

The healing products covering the cracks were identified as the reaction products of healing agent released from microcapsules and the host matrix. This material was considered to behave as a scaffold for the proliferation of autogenic healing material.

This study has showed that undoubtedly microencapsulated silica precursors can be successfully used for autonomic healing of cement-based materials. Mineral core materials 
provide greater affinity and allow for extended shelf-life which would translate to long term solution in situ. The main potential of the proposed systems is identified in the durability recovery, reduction of permeability and diffusion of external degrading agents. The next step has been taken towards commercialization of the proposed solution with full scale field applications with a trial structures at the Heads of the Valleys highway project in South Wales [14] and as part of extension work of the main Engineering Department in Cambridge (Dyson Building) [15] to establish the real time efficacy in infrastructure and identify any potential limitations during processing.

\section{ACKNOWLEDGEMENTS}

Financial support from the Engineering and Physical Sciences Research Council (EPSRC) for this study (Project Ref. EP/K026631/1 "Materials for Life") is gratefully acknowledged.

\section{REFERENCES}

[1] White SR, Sottos NR, Geubelle PH, Moore JS, Kessler MR, Sriram SR, et al. Autonomic healing of polymer composites. Nature 2001;409:794-7.

[2] Dry C. Matrix cracking repair and filling using active and passive modes for smart timed release of chemicals from fibers into cement matrices. Smart Mater Struct 1994;3:118-23.

[3] Pelletier M, Bose A. Self-mending composites incorporating encapsulated mending agents. US20110316189 A1, 2011.

[4] Yang Z, Hollar J, He X, Shi X. A selfhealing cementitious composite using oil core/silica gel shell microcapsules. Cem Concr Compos 2011;33:506-12.

[5] Su JF, Qiu J, Schlangen E. Stability investigation of self-healing microcapsules containing rejuvenator for bitumen. Polym Degrad Stab 2013;98:1205-15.
[6] Lark RJ, Al-Tabbaa A, Paine K. Biomimetic multi-scale damage immunity for construction materials : M4L Project overview. 4th Int. Conf. Self-Healing Mater., 2013, p. 2-5.

[7] Kanellopoulos A, Qureshi TS, A1Tabbaa A. Glass encapsulated minerals for self-healing in cement based composites. Constr Build Mater 2015;98:780-91.

[8] Qureshi T, Kanellopoulos A, Al-Tabbaa A. Encapsulation of expansive powder minerals within a concentric glass capsule system for self-healing concrete. Constr Build Mater 2016; In Press.

[9] Wang JY, Soens H, Verstraete W, De Belie N. Self-healing concrete by use of microencapsulated bacterial spores. Cem Concr Res 2014;56:139-52.

[10] Giannaros P, Kanellopoulos A, AlTabbaa A. Sealing of cracks in cement using microencapsulated sodium silicate. Smar 2016; Paper acce.

[11] Gilford III J, Hassan MM, Rupnow T, Barbato M, Okeil A, Asadi S. Dicyclopentadiene and Sodium Silicate Microencapsulation for Self-Healing of Concrete. J Mater Civ Eng 2014;26:88696.

[12] Mostavi E, Asadi S, Hassan MM, Alansari M. Evaluation of Self-Healing Mechanisms in Concrete with DoubleWalled Sodium Silicate Microcapsules. J Mater Civ Eng 2015:04015035.

[13] Brown EN, White SR, Sottos NR. Microcapsule induced toughening in a self-healing polymer composite. J Mater Sci 2004;39:1703-10.

[14] BBC. "Self-healing" concrete trial launched in south Wales - BBC News 2015.

[15] Cardno CA. Concrete Test Blocks Top "Living Building"| ASCE. ASCE - Civ Eng 2015. 